\title{
Investigation of void fraction schemes for use with CFD-DEM simulations of fluidized beds
}

\author{
Daniel A. Clarke, ${ }^{\dagger}$ Andrew J. Sederman, \\ Lynn F. Gladden, ${ }^{\ddagger}$ and Daniel J. Holland ${ }^{*}{ }^{\dagger}$ \\ ${ }^{\dagger}$ Department of Chemical and Process Engineering, University of Canterbury, \\ Private Bag 4800, Christchurch 8140, New Zealand \\ Department of Chemical Engineering and Biotechnology, University of Cambridge, Cambridge \\ University West Site, Philippa Fawcett Dr, Cambridge CB3 0AS, United Kingdom
}

Corresponding Author*: daniel.holland@,canterbury.ac.nz

Keywords: CFD-DEM, fluidization, void fraction, MRI, numerical simulation 


\begin{abstract}
This paper investigates the spatial resolution of computational fluid dynamics-discrete element method (CFD-DEM) simulations of a bubbling fluidized bed for seven different void fraction schemes. Fluid grids with cell sizes of 3.5, 1.6 and 1.3 particle diameters were compared. The particle velocity maps from all of the void fraction schemes were in good qualitative agreement with the experimental data collected using magnetic resonance imaging (MRI). Refining the fluid grid improved the quantitative agreement due to a more accurate representation of flow near the gas distributor. The approach proposed by Khawaja et al. (J. Comput. Multiphase Flows, 2012, 4, 183-192) provided the closest match to the exact void fraction though only the particle centered method differed significantly. These results indicate that the fluid grid used for CFDDEM simulations must be sufficiently fine to represent the inlet flow realistically, and that a void fraction scheme such as that proposed by Khawaja be used.
\end{abstract}




\section{INTRODUCTION}

Gas-solid fluidized beds exhibit complicated, scale dependent flow behavior which makes designing industrial scale equipment challenging ${ }^{1}$. Computational fluid dynamics coupled with discrete element modelling (CFD-DEM) is part of the multiscale modeling framework. In this framework, highly detailed simulations that are only viable for systems sized at the laboratory scale are used to provide phenomenological insight and to develop closure relations for numerical methods that are suitable on industrial length scales. These latter models are then used to aid process design ${ }^{2}$. CFD and DEM are coupled by calculating the localized proportion of volume occupied by the gas phase, referred to here as the void fraction, or voidage. This calculation is handled by a void fraction scheme. The void fraction within a fluid cell may be determined exactly ${ }^{3}$. However, such a calculation may require a complicated mathematical model and considerable computational expense, especially for complex geometries, nonspherical particles, and unstructured CFD grids. As a result, non-analytical approximations to the void fraction have been used to simulate interacting fluid-particle flows. These void fraction schemes are then applied to each cell in the fluid grid. CFD-DEM models require careful balancing of the cell sizes used in the fluid model. If the cells are too coarse key features of the fluid flow field will be missed, on the other hand if the cells are too fine the volume averaging assumptions used to derive the fluid dynamic equations may break down ${ }^{4-6}$. The approximations made by void fraction schemes may alter the minimum acceptable cell size for the fluid dynamics model ${ }^{5}$. Here several of the most common void fraction schemes were used to simulate the fluidization above a drilled plate distributor where a refined fluid grid was required.

The CFD-DEM approach is based on volume averaging of the gas and particulate phases ${ }^{4}$. In these equations an assumption is made that the length scale over which the fluid dynamics 
changes is large compared with the length scale of the particles. This so called "separation of scales" is what permits the use of a simple drag force correlation to describe the fluid-particle interaction. Many studies simply assume that a minimum cell size of $\sim 3$ particle diameters $\left(d_{p}\right)$ is sufficient for the volume averaging equations to be valid. However, in a detailed study Peng et al. ${ }^{5}$ demonstrated by analysis of bubble evolution and pressure fluctuations that cell sizes as small as about $1.6 d_{p}$ may be sufficient for the assumption of a separation of scales to be valid. By contrast, Boyce et al. ${ }^{6}$ found that a minimum cell size of $\sim 3 d_{p}$ was required to accurately simulate the frequency of bubble eruptions and pressure fluctuations. Therefore, there is still some debate in the literature as to the minimum cell size that can be used in CFD-DEM simulations. In the work of Peng et al. ${ }^{5}$, the method used to calculate the void fraction was critical in establishing the minimum feasible cell size. Here we investigate these methods further.

A void fraction scheme should (i) conserve the total mass of the solid phase, (ii) predict a gridindependent velocity field, and (iii) produce smooth void fraction fields within the densely packed particle phase ${ }^{7}$. If these conditions are not met, then the local void fraction may be unrealistic (i.e. below the close packing limit or greater than unity). It is then likely that the value of the local drag force would also be unrealistic. An unreasonably high (or low) local drag force may give an unstable solution to the fluid phase system of equations. The solution may also be unstable if the time derivative of the local void fraction were to become unrealistically large ${ }^{5}$. This result may occur if a small change in the positions of the particles results in a large change in the local void fraction in a cell. Several void fraction schemes have been devised to attempt to fulfil these three criteria.

The simplest void fraction scheme is the particle-centered method (PCM), which assumes that the entire particle volume is located at the centroid of the particle ${ }^{8}$. Void fraction fields in 
simulations using the PCM display large fluctuations with time due to particle centers crossing cell boundaries ${ }^{9}$. If the cell size to particle diameter ratio is greater than 3.8 , then unrealistic spikes in the local gas pressure do not occur. Thus, the PCM approach is likely satisfactory if fluid cells are large compared to the particle size. However, in order to capture the fluid flow field, especially near surfaces, it is often desirable to use cells that are not much larger than the diameter of the particles where the PCM approach is likely invalid.

Exact void fractions can be obtained analytically ${ }^{3}$ and quickly, but this analytical approach is only practicable if using a simple cuboidal mesh. For more complex meshes, the intersecting volumes between each particle and the local mesh elements can be determined by decomposing the sphere into a collection of spherical caps, wedges and cones ${ }^{10}$. In theory, a typical system comprising $\sim 10^{5}$ particles would require $\sim 10$ seconds to calculate all of the potential overlap volumes for all of the particles in all 27 neighboring cells (in a three-dimensional system), several orders of magnitude longer than the cuboidal exact method. Several methods have been proposed to strike a balance between computational efficiency and accuracy of voidage calculations.

The first methods we consider are the so called "divided particle volume methods" (DPVMs) in which the shape of the particle is approximated by some simple geometric approach, such as a cube. Portions of the volume of the particle are then assigned to several nearby cells. One example of this approach is used in the MFIX software ${ }^{11-13}$.

A second approach, the statistical kernel methods, determine the solid volume fraction by distributing the particle volume by a weighting function that decays away from the particle center $^{14}$. The Gaussian distribution is often used as a weighting function and hence a particle may contribute a portion of its volume to distant cells. In order to execute the statistical kernel 
method with distributed memory parallel systems a PDE for the diffusion of the void fraction may be solved over a series of pseudo-time steps ${ }^{7}$. With the correct initialization, the diffusion method is equivalent to the statistical kernel method, and is faster to implement.

Another approach that has been proposed is to sub-divide the particle into a collection of smaller entities. The volume fraction of the particle may then be determined by the amount of these entities within each cell. Here, such methods are denoted as "satellite point methods"15-18. This method was used by Hobbs ${ }^{19}$ to estimate the void fraction for non-rectangular cells in an unstructured grid without strenuous mathematical effort.

Two-grid methods consist of a dedicated particle grid and a dedicated fluid grid. The void fraction is calculated on the particle grid, which is usually taken to be a Cartesian grid. The void fraction on the Cartesian grid may be calculated using the PCM, or any other suitable scheme. This void fraction data is then mapped onto a fluid grid where the fluid phase conservation equations are solved. The two-grid method has been used to map void fraction data to unstructured or non-Cartesian grids where other void fraction calculations are more difficult to implement ${ }^{20}$. In this case, the volume fraction of each fluid cell in each particle cell only needs to be determined at the initialization stage, reducing the computational expense ${ }^{20}$.

The purpose of this study was to investigate the different void fraction schemes used with CFD-DEM when simulating a flow field with features that occur on a length scale comparable to the length scale of the particles. Seven different void fraction schemes were tested, with the specific methods used outlined in Section 2. Simulations were performed of a fluidized bed with a drilled plate distributor. The holes in the drilled plate were $1 \mathrm{~mm}$ diameter, while the particles simulated were $1.07 \mathrm{~mm}$ in diameter, thus some features of the fluid flow field will occur over a length scale comparable to the size of the particles. Here simulations of the fluidization were 
performed with cell sizes of $3.7 \mathrm{~mm}, 1.8 \mathrm{~mm}$, and $1.4 \mathrm{~mm}$. Simulation results were validated by comparison with experimental measurements performed by Magnetic Resonance Imaging (MRI). This investigation builds upon the work in this field by comparing the non-analytical methods in order to ascertain the efficacy of these methods when simulating a high-resolution flow field.

\section{METHOD}

\subsection{EXPERIMENT}

The data were collected from a square fluidized bed, with cross-sectional dimensions of $37 \mathrm{~mm} \times 37 \mathrm{~mm}$ and with a settled bed height of $30 \mathrm{~mm}$. Humid air at a pressure of 1 barg was used as the fluidizing gas. The gas distributor was a drilled plate with a $4 \times 4$ grid of $1 \mathrm{~mm}$ diameter holes, each spaced $7 \mathrm{~mm}$ apart. The pressure drop across the distributor exceeded the pressure drop across the bed at minimum fluidization, thus ensuring even gas distribution. Air flow near the walls was restricted since the holes were all located inside a $23 \mathrm{~mm}$ wide square region at the center of the distributor. The time-averaged particle velocity throughout the bed was measured using MRI. Rhoeas poppy seeds were used as particles, since their nuclear-spin relaxation properties were advantageous for imaging. The seeds were kidney shaped and had a mean projected area-equivalent diameter of $1.07 \mathrm{~mm}$ and a measured minimum fluidization velocity of $0.3 \mathrm{~m} / \mathrm{s}$ at STP. The superficial gas velocity was equal to $0.6 \mathrm{~m} / \mathrm{s}$.

The MRI experiments were conducted with a Bruker DMX 200 spectrometer operating in the vertical direction, for which the proton $\left({ }^{1} \mathrm{H}\right)$ frequency was $199.7 \mathrm{MHz}$. The seeds were excited and detected using a birdcage radio frequency coil surrounding the fluidized bed. A 3-axis shielded gradient system was used to establish spatial resolution, with a maximum magnetic field 
gradient strength of $0.139 \mathrm{~T} / \mathrm{m}$. A Cartesian coordinate system, with the $z$-axis pointing along the vertical direction was used. The velocity of the particles was measured using pulsed field gradient MRI as is described in detail elsewhere ${ }^{21}$. In brief, a pair of half sine-shaped motion encoding gradients were used to encode the velocity in the phase of the observed signal. The gradients were each applied for a half-period $(\delta)$ of $0.56 \mathrm{~ms}$ and the observation time between gradients $(\Delta)$ was $2 \mathrm{~ms}$. The amplitude of the sine gradient $(g)$ was adjusted in 10 steps about 0 $\mathrm{T} / \mathrm{m}$, with a maximum gradient strength of $0.11 \mathrm{~T} / \mathrm{m}$. A time-averaged image of the velocity was obtained by collecting 48 averages over a total time of 2 hours.

The local mean velocity was obtained by the gradient of the linear equation fitted to the plot of the angle of the complex signal against the inverse variable $\mathbf{p}=\gamma g \delta \Delta$. The uncertainty of the measurement was estimated from the error of the fitted line. In areas of low velocity, the uncertainty was $\pm 0.002 \mathrm{~m} / \mathrm{s}$. In regions of high velocity and low signal-to-noise, the error increased but was $<0.02 \mathrm{~m} / \mathrm{s}$ for $95 \%$ of the voxels. For simplicity, a constant margin of \pm 0.02 $\mathrm{m} / \mathrm{s}$ was used to denote the uncertainty in the experimental velocity profiles.

\subsection{SIMULATION SETUP}

The open-source CFD-DEM package in MFIX was used to run the simulations in this work. The particle motions were modelled using the soft-sphere DEM, while the fluid flow was resolved by solving the volume-averaged mass and momentum conservation equations using an extension of the SIMPLE method. Full details of the computational model are provided by Syamlal et al. ${ }^{22}$; the main equations of the model are included in the Supporting Information. Verification $^{23-25}$ and validation ${ }^{26,27}$ of MFIX has been performed, demonstrating that the models have been correctly implemented and are physically meaningful. The MFIX source code was 
modified to add the void fraction schemes that were not incorporated into the standard MFIX package. The simulated domain was a square fluidized bed, with dimensions of $37 \mathrm{~mm} \times 37 \mathrm{~mm}$ $\times 120 \mathrm{~mm}$. The square grid configuration avoids complications arising from curved boundaries and also simplifies the implementation of the void fraction codes since they did not need to consider non-rectangular cells. Three grid resolutions were tested: $10 \times 10 \times 32$ cells (cell length to particle diameter ratio $=3.5), 21 \times 21 \times 68$ cells $($ cell length to particle diameter ratio $=1.6$ ), and $26 \times 26 \times 84$ cells (cell length to particle diameter ratio $=1.3$ ). Here these grids are referred to as coarse, fine and very fine respectively. The cell length to particle diameter ratios were greater than the minimum recommended value of $1.63^{5}$ for the coarse and fine grids, but below this threshold for the very fine grid. The dimensions of the fluid domain and the settled particle bed are given by Figure 1(a).

A constant-mass inflow boundary condition was applied at the inlet. The coarse grid was not able to resolve the gas flow through the holes since the hole diameter was about one quarter of the cell length. Thus, a uniform boundary was applied to the central $33 \mathrm{~mm} \times 33 \mathrm{~mm}$ region to provide the best approximation to the distributor geometry. The velocity in this region was set to $0.754 \mathrm{~m} / \mathrm{s}$, while the gas velocity in the outer perimeter was set to zero. This arrangement gave the same total mass flow rate of gas as in the experiments. For the fine grid, the gas velocity was set to $16.54 \mathrm{~m} / \mathrm{s}$ in 16 cells, each representing a hole in the distributor. The air velocities into the remaining cells at the distributor were set to zero. This level of grid refinement was close to the minimum cell size to particle diameter ratio at which the separation of scales assumption was found to be valid ${ }^{5}$. For the very fine grid, the gas velocity was set to $25.35 \mathrm{~m} / \mathrm{s}$ in 16 cells. This grid is below the recommended minimum cell size to particle diameter ratio. Figure 1 shows the configurations of the inlets. The outlet boundary condition was set to constant-pressure outflow. 
A no-slip boundary condition was applied at the wall for the gas phase. Müller et al. ${ }^{28}$ found that the choice of wall boundary condition did not affect the void fraction profile. Ye et al. ${ }^{29}$ found that for Group A particles, the no-slip boundary condition predicted a minimum bubbling velocity that was considerably higher than the experimental value. In contrast, the full-slip boundary condition predicted a minimum bubbling velocity that was slightly lower than the value obtained experimentally. In our case, analysis of averaged particle phase velocity maps suggested that the choice of gas phase wall boundary condition did not have a discernible impact upon the particle trajectories. Thus, the selection of the no-slip boundary condition was arbitrary.

The linear-spring dashpot model was used to calculate the contact forces during particleparticle and particle-wall interactions. The Beetstra model ${ }^{30}$ was used to calculate the drag force acting on the particles. The main parameters that were used in all the simulations are given in Table S1 (in the Supporting Information). The ratio between the tangential and normal spring constants was equal to $2 / 7$ to equalize the periods of normal and tangential contact oscillations $^{31,32}$. The DEM time step was $1.15 \times 10^{-6} \mathrm{~s}$, or $1 / 50^{\text {th }}$ of the minimum collision time ${ }^{33}$. The local relative velocity distribution of colliding particles (shown in Figure S1 in the Supporting Information) showed that the relative velocity of all contacts was below $0.06 \mathrm{~m} / \mathrm{s}$. For the contact parameters used, the maximum overlap between particles for a collision with a relative velocity of $0.06 \mathrm{~m} / \mathrm{s}$ was $0.1 \%$, well below the threshold of $1 \%$ that is required to ensure that rigid particle contacts are well approximated ${ }^{34}$. The fluid time step was adaptive. If the solution to the fluid phase conservation equations did not converge at a particular time step, the time step value was reduced. The maximum time step that was allowed was $5 \times 10^{-4} \mathrm{~s}$. This time step was sufficient to achieve convergence in the coarse grid case. For the fine grid, the time step was usually between $1 \times 10^{-4}$ and $2 \times 10^{-4}$ s owing to the reduction of the cell size. The simulations 
were weakly coupled; up to 400 time steps of the DEM were run between each fluid phase time step. In coarse grid simulations using the PCM, it was found that reducing the fluid phase time step to as little as $5 \times 10^{-6} \mathrm{~s}$, or $\sim 5$ DEM time steps, did not quantitatively influence the timeaveraged particle velocity distribution. Thus, an upper limit of $5 \times 10^{-4} \mathrm{~s}$ was used in subsequent simulations in order to reduce the total computation time.

Each simulation was run in serial mode, using an Amazon Web Services EC2 virtual computing instance. The instance consisted of one thread of an Intel Xeon E5-2670 v2 CPU, with $3.75 \mathrm{~GB}$ of memory and a clock frequency of $2.5 \mathrm{GHz}$. Each simulation was run to simulate $5 \mathrm{~s}$ of operation. A simulation of this length took approximately 36 hours to complete for the coarse grid and 150 hours for the fine grid. The profiles of the pressure drop against time (shown in Figure S2 in the Supporting Information) demonstrate that the pressure fluctuates steadily after $1 \mathrm{~s}$ of simulated time. The standard deviation of pressure fluctuations calculated over intervals of $1 \mathrm{~s}$ was constant between $2 \mathrm{~s}$ and $5 \mathrm{~s}$. For these reasons, data collected beyond $2 \mathrm{~s}$ was used for averaging.

The time required for the calculations associated with the void fraction schemes was an area of interest. If the void fraction code takes less time to execute, then the total simulation time may be reduced, thus minimizing the computational resources required. However, it is also possible that temporal fluctuations in the void fraction may impede the convergence of the fluid phase solver. To assess these competing effects, the total CPU time for each coarse grid simulation was recorded from the output files. Additionally, the CPU time dedicated to calculating the void fraction itself was estimated by calculating the time taken to run the relevant subroutines. For the void fraction timing tests, the void fraction time was sampled for $0.01 \mathrm{~s}$ of operation (about 20 iterations for the coarse grid case) for each of the simulations. 


\subsection{IMPLEMENTATION OF VOID FRACTION SCHEMES}

Figure 2 gives visual illustrations of the analytical, PCM, MFIX DPVM, cube DPVM, corrected cube DPVM, statistical kernel and satellite point methods. Since the two-grid method is not completely distinct from the other void fraction models when used with regular Cartesian fluid cells, it will not be investigated in this study. The left-hand diagrams illustrate where the particle is positioned on the fluid grid. Visual representations of each void fraction scheme are included to illustrate how the particle volume fraction in each cell is calculated. The right-hand images describe the spatial distribution of the particle volume fraction, where high particle volume fraction is denoted with dark cells. The formulation of each void fraction scheme and the values of the relevant parameters are described in the following sections:

\section{PCM}

The void fraction of a fluid cell using the PCM is given by:

$$
\varepsilon_{g}(\boldsymbol{x})=1-\frac{\sum_{p=1}^{N_{\text {particles }}} V_{p}}{V(\boldsymbol{x})}
$$

where $\varepsilon_{g}(\boldsymbol{x})$ is the local gas phase volume fraction at position $\boldsymbol{x}, V_{p}$ is the volume of particle $p$ and $V(\boldsymbol{x})$ is the volume of the cell located at position $\boldsymbol{x} . N_{\text {particles }}$ is the number of particle centers located inside the cell.

\section{MFIX DPVM}

The MFIX DPVM estimated the void fraction in a cell by:

$$
\varepsilon_{g}(\boldsymbol{x})=1-\frac{\sum_{p=1}^{N_{\text {particles }}} \phi_{p}(\boldsymbol{x}) V_{p}}{V(\boldsymbol{x})}
$$


where $\phi$ is the volume fraction of particle $p$ within the cell located at position $\boldsymbol{x}$. Particles with centers in the cell of interest, or in the 26 neighboring cells may have a non-zero value of $\phi$ with respect to the cell at position $\boldsymbol{x}$. Therefore, $N_{\text {particles }}$ is the number of particles with centers located in the cell of interest and in its immediate neighbours.

Firstly, two weightings were calculated for each dimension, $\phi_{k, F-}$ located at the cell face before the central co-ordinate and $\phi_{k, F+}$ located at the cell face after the central co-ordinate. The values of these weightings in direction $k$ were given by:

$$
\phi_{k, F}=\left\{\begin{array}{cl}
\frac{h^{2}(3 w-h)}{4 w^{3}} & , \text { if } \delta x_{p, F}<w \\
0 & , \text { if } \delta x_{p, F} \geq w
\end{array}\right.
$$

where $w$ is the filter width, $\delta x_{p, F}$ is the distance between the particle center and the face $F$ and $h=w-\delta x_{p, F}$. The filter width used for this investigation was equal to the particle diameter, $1.07 \mathrm{~mm}$. Using these two weightings, the weighting along direction $k$ for the cell in which the particle is located was calculated from:

$$
\phi_{k}=1-\phi_{k, F+}-\phi_{k, F-}
$$

The weighting for neighboring cells is given by $\phi_{k, F}$. For example, in the $x$-direction, $\phi_{x, F-}$ and $\phi_{x, F+}$ would be the weightings obtained using the overlap between the $x$-component of the particle center and the western and eastern faces respectively. If $h$ is greater than the filter width, then $\phi_{k, F}$ is set to zero. Provided that $w$ is smaller than the dimension of the cell, either, $\phi_{k, F-}$ or $\phi_{k, F+}$ will be zero. Subsequently, the volume fraction for particle $p$ in a cell is the product of the weightings calculated in each dimension:

$$
\phi_{p}(\boldsymbol{x})=\phi_{x}(\boldsymbol{x}) \phi_{y}(\boldsymbol{x}) \phi_{z}(\boldsymbol{x})
$$

The overall void fraction in a cell is then calculated from Equation 2. 


\section{Cube DPVM}

Like the MFIX DPVM, the cube DPVM determined the void fraction using Equation 2. However, the volume fraction of particle $p$ was estimated differently. $\phi_{p}$ was determined by:

$$
\phi_{p}(x)=\frac{\delta x \delta y \delta z}{D_{p}^{3}}
$$

where $\delta x, \delta y$, and $\delta z$ are the lengths of the cuboid that contains the portion of the particle that lies within the cell. The cuboid length in direction $k$ is calculated from:

$$
\delta x_{k}=\min \left(x_{k, F+},\left(x_{p, k}+r_{p}\right)\right)-\max \left(x_{k, F-},\left(x_{p, k}-r_{p}\right)\right)
$$

where $x_{p, k}$ is the position of particle $p$ in direction $k, x_{k, F+}$ is the position of the eastern, northern or top face in direction $k$ and $x_{k, F-}$ is the position of the western, southern or bottom face.

\section{Corrected Cube DPVM}

The cube DPVM approach is attractive as it is simple to implement and computationally efficient. However, Khawaja et al. ${ }^{13}$ found that the volume fraction estimated using the cube DPVM under-estimated the true volume fraction when there was only a small amount of the particle outside of the cell. In order to improve the approximation of the particle volume fractions, they developed an equation to convert the cube volume fractions to values that were in closer agreement with the volume fraction obtained for a sphere analytically. Their correlation is given by:

$$
\phi_{p}(\boldsymbol{x})=-0.8457 \phi_{\text {cube }}(\boldsymbol{x})^{3}+1.6625 \phi_{\text {cube }}(\boldsymbol{x})^{2}+0.1832 \phi_{\text {cube }}(\boldsymbol{x})
$$

where $\phi_{\text {cube }}$ was determined by Equations 6 and 7. For each particle, it was unlikely that the values of $\phi_{p}$ over all the cells in which the particle resided would sum to unity. Thus to ensure 
conservation of mass, after $\phi_{p}$ was calculated, it was normalized such that $\sum \phi_{p}=1$, where the sum is performed over all cells in which the particle may reside. Equation 2 was then applied to determine the void fraction.

\section{Statistical Kernel/Diffusion Method}

The statistical kernel method determines the volume fraction of a particle in a cell by using a weighting function. Weighting functions that have been used in previous investigations include the Gaussian distribution ${ }^{35}$ and the distribution function of Johnson ${ }^{14,36}$. The region over which the volume of a particle is distributed may exceed the boundaries of the domain. The portion of the weighting function outside of the domain is discarded. In order to conserve the total solid volume, a ghost particle is placed outside the domain, mirroring the location of the original particle. The weighting functions of both particles are combined. The integral of the new weighting function within the domain is equal to unity, thus the particle volume is conserved.

Here the statistical kernel method was approximated using the diffusion approach ${ }^{7}$. The diffusion of the void fraction may be described by:

$$
\frac{\partial \varepsilon_{g}}{\partial t_{d}}=\nabla^{2} \varepsilon_{g}
$$

where $\varepsilon_{g}$ is the void fraction and $t_{d}$ represents the pseudo-time domain over which the diffusion takes place. Equation 9 was discretized using the finite volume method with a central differencing scheme. The PCM solid volume fraction was used as the initial condition. The system of linear equations was solved to update the void fraction field at the next pseudo-time step. For each void fraction calculation, 5 pseudo-time steps were used. With 5 pseudo-time steps, the voidage distribution was approximately Gaussian but somewhat narrower than expected based on the specified diffusion time. Increasing the number of steps resulted in a 
distribution that was closer to Gaussian, but this was not felt to improve the results. The volume fraction of each particle in a cell using the statistical kernel method depends on the distance between the particle and cell centers. The particle volume fraction does not depend on this displacement for the diffusive method; hence it does not contain information about particle locations at the sub-grid scale. Therefore, the voidage calculated by the diffusive method is not, in general, identical to the statistical kernel method. However, if the diffusion length (or standard deviation) is sufficiently large the difference between the statistical kernel method and the diffusive method is negligible.

The analysis was performed using pseudo-time steps of $2.28 \times 10^{-7} \mathrm{~s}$ and $9.16 \times 10^{-7} \mathrm{~s}$. The diffused voidage profiles for a single particle resembled a distribution where the standard deviation was equal to $1 d_{p}$ and $2 d_{p}$ respectively.

\section{Satellite Point Method}

The satellite point method approximates a particle as a cluster of smaller pseudo-particles. The pseudo-particles are distributed at different positions within the particle. The volume fraction of the particle within a particular cell is estimated by:

$$
\phi(\boldsymbol{x})=\frac{N_{p}(\boldsymbol{x})}{N_{p, \text { total }}}
$$

where $N_{p}(\boldsymbol{x})$ is the number of pseudo-particles located inside the cell located at position $\boldsymbol{x}$. $N_{p, t o t a l}$ represents the total number of pseudo-particles that make up a real particle.

The code that was developed to use this scheme in MFIX distributed pseudo particles at the same locations as the CFDEM-Coupling scheme ${ }^{37}$. One pseudo-particle was at the center of the real particle. Two rings, with elevation angles of $\frac{\pi}{4}$ and $\frac{3 \pi}{4}$ and each with 4 pseudo-particles were distributed around the center of the real particle. The pseudo-particles in each of these rings had 
a radial coordinate of $0.62 r$ and azimuth angles of $\frac{\pi}{4}, \frac{3 \pi}{4}, \frac{5 \pi}{4}$ and $\frac{7 \pi}{4}$. There were a further 6 particles located $0.62 r$ away from the center and positioned along the positive and negative directions of the $x$-, $y$ - and $z$-axes. This arrangement was repeated for another 14 pseudo-particles at a radial coordinate of $0.92 r$. In total, one particle was represented by 29 pseudo-particles. This arrangement of pseudo-particles means that each pseudo-particle represented approximately the same volume of the real particle. For the coarse grid, Peng et al. ${ }^{16}$ recommend a minimum number of satellite points to be 5 and advise using at least five times this number for a realistic solution. Hence, the prediction accuracy of the satellite point method as implemented was deemed to be adequate.

The most simplistic approach for binning particles to the fluid grid was to search for each satellite point, which was computationally slow. Advanced search algorithms have been developed to improve the efficiency of this process ${ }^{16,38}$. The gain in computation speed was marginal for the simulations in this study due to the weak coupling between the fluid and particle phases. Hence, the location of each satellite point was found individually.

\subsection{POST-PROCESSING AND ANALYSIS}

For the purpose of averaging, only the last $3 \mathrm{~s}$ of data was used to minimize any start-up effects that may have occurred. Averaging was necessary to facilitate a fair comparison between each set of results and the experimental data. The particle-weighted time-averaged solid velocity was used since MR images correspond to a particle-based time average ${ }^{35}$ and was calculated by:

$$
\boldsymbol{v}_{s, \text { avg }}(\boldsymbol{x})=\frac{\sum_{t=1}^{N_{\text {frames }}} \sum_{p=1}^{N_{\text {particles }}(\boldsymbol{x})} \boldsymbol{v}_{p, t} \phi_{p, t}(\boldsymbol{x})}{\sum_{t=1}^{N_{\text {frames }}} \sum_{p=1}^{N_{\text {particles }}(\boldsymbol{x})} \phi_{p, t}(\boldsymbol{x})}
$$


where $\boldsymbol{v}_{p, t}$ is the velocity of particle $p$ at time $t$ and $\phi_{p, t}(\boldsymbol{x})$ represents the volume fraction of particle $p$ inside the voxel $\boldsymbol{x}$ at time $t$. The volume fraction was determined using the corrected cube DPVM of Khawaja et al. ${ }^{13}$ irrespective of which void fraction scheme was used during the simulation itself. This method was used since it was able to give accurate estimations of the volume fractions, while still being efficient to implement computationally. The PCM was considered as well, however it was not able to give representative averages of the particle velocity for voxels near the domain boundary due to ordering of the particles near the walls.

The relative discrepancy between two fields was calculated using:

$$
\Delta \zeta=\frac{\sum\left(\zeta^{A}(\boldsymbol{x})-\zeta^{B}(\boldsymbol{x})\right)^{2}}{\sum \zeta^{B}(\boldsymbol{x})^{2}} \times 100 \%
$$

where $\zeta$ may be either the void fraction or the particle phase velocity. For comparisons with the experiments, the simulation data took the place of $\zeta^{A}(\boldsymbol{x})$ and the experimental particle velocity field took the place of $\zeta^{B}(\boldsymbol{x})$. Simulation data were also compared with other simulation data with the assignment to $\zeta^{A}(\boldsymbol{x})$ and $\zeta^{B}(\boldsymbol{x})$ detailed in the results.

\section{RESULTS AND DISCUSSION}

\subsection{EVALUATION OF VOID FRACTION SCHEMES}

In order to identify and understand the differences in the void fraction schemes, each scheme was applied to a set of particles' positions selected from a simulation. Two points in time that were $0.01 \mathrm{~s}$ apart were used in order to identify any large temporal changes in the local void fraction. The coarse grid $(10 \times 10 \times 32$ cells $)$ and the fine grid $(21 \times 21 \times 68$ cells $)$ were used. The void fraction maps using the coarse grid are given in Figure 3 and the fine grid in Figure 4. The thickness of the sample region was equal to one fluid cell. Using the metrics of Peng et al. ${ }^{5}$, the ratio between the effective domain length and the effective cell length, $S_{d} / S_{c}$ for the coarse 
grid was equal to 14.7 and the fine grid 31.1 . Thus, $S_{d} / S_{c}$ was below the minimum recommended value of 19.3 for the coarse grid, which implied that the solution may be influenced by the resolution of the fluid grid. For each set of void fraction maps, the right-hand map was made using particle positions $0.01 \mathrm{~s}$ after those used to make the left-hand map. The exact void fraction was calculated using the method and code developed by Strobl et al. ${ }^{10}$. Using this method required a computation time of $\sim 1 \mathrm{~s}$, which was faster than estimated by Strobl et al. ${ }^{10}$, and slower than the non-exact methods. With the exception of the fine grid PCM and $2 d_{p}$ diffusive scheme, all of the void fraction schemes predicted void fraction fields that were visually similar to the exact void fraction field. The relative deviations obtained using each void fraction scheme and the exact void fraction field (shown in Table S2 in the Supporting Information) and were consistently low. However, the relative discrepancy values differed over several orders of magnitude, ranging from $\sim 10^{-5} \%$ for the corrected cube DPVM, which was the most accurate technique, to $\sim 10^{-1} \%$ for the diffusive scheme which was the least accurate technique. The large deviation was due to the spatial smoothing which blurred out local features of the voidage maps.

The relative discrepancy was also affected by the resolution of the void fraction map. The PCM was found to be unsuitable for the fine grid since there were cells which had a negative void fraction due to too many particle centers being located within single cells. Increasing the grid refinement increased the relative discrepancy of all schemes by between a factor of 2 (diffusive) and a factor of 20 (PCM). The amplification in this total error was because more particles are located within several cells as the grid was refined; hence the errors introduced by each voidage scheme are more numerous. Furthermore, the relative error in the local volume fraction is increased by the reduction in the cell volume. Since the void fraction maps for all 
schemes were similar, fine grid simulations were only performed using the corrected cube and the diffusive ( $\left.\sigma=1 d_{p}\right)$ schemes in further studies; coarse grid simulations were performed for all schemes.

\subsection{ANALYSIS OF COMPUTATION TIME}

Details of the computation time of the void fraction schemes are provided in Table S1 in the Supporting Information. For the coarse grid, there was no clear relation between the times required to run the full simulations and the times required to calculate the void fraction. This result was not surprising since the simulations had weak gas-solid coupling. For the PCM, the total time spent calculating the void fraction was $0.02 \%$ of the total CPU time. Even for the satellite point method, the void fraction calculations accounted for only $1.21 \%$ of the total CPU time. In these simulations other processes would have had a larger influence on the total computational expense. For example, the contact forces between particles were another calculation that was computationally intensive. If there were a high number of particle pairs in contact, then the simulation would require more time to run than if there were a lower number of contacting pairs. When the bed was expanded, there were fewer contacts to resolve. The bed did not expand as much with the diffusive scheme $\left(\sigma=2 d_{p}\right)$ compared to other void fraction schemes. Hence, the simulation using the diffusive scheme may have had to handle more particle contacts, leading to an increase in the simulation time overall, even though the time required to evaluate the void fraction calculation itself was short. Thus, the choice of void fraction scheme did not drastically increase the computational time, provided that the particle and fluid phases were weakly coupled and that all simulations use the same time step value.

For the fine grid simulations, the corrected cube DPVM required about the same amount of time to calculate the void fraction as for the coarse grid case. This was expected since the 
algorithm only considered the immediate neighbors of the cell in which the particles are centered, and hence scales with the number of particles and is not strongly influenced by the number of fluid grid cells. The diffusive method took considerably longer since the diffusion equation had to be solved for more cells compared to the coarse grid case. However, the diffusive method simulation had a much shorter run time overall, due to fewer fluid steps being performed (hence a longer mean time step) compared to the corrected cube simulation. The variance of the void fraction spatial gradients was around $1100 \mathrm{~m}^{-2}$ for the corrected cube method

and $700 \mathrm{~m}^{-2}$ for the diffusive scheme. The means of the absolute normalized net gas mass flow rate were $0.84 \%$ for the corrected cube method and $0.58 \%$ for the diffusive scheme. These metrics suggested that the diffusive scheme offered superior mass conservation and spatial smoothing, allowing the solution to the system of equations to converge at a longer time step and hence reducing the overall computation time by about $15 \%$. As an extreme case, the PCM was unstable using the fine grid. Thus, the choice of void fraction scheme had a noticeable effect upon the numerical stability for the refined simulations, but not for the coarse grid simulations.

\subsection{COMPARISON WITH EXPERIMENT}

The maps for the time-averaged vertical particle velocity along horizontal and vertical slices obtained using MRI were used as a reference against which the images produced from the simulation data were compared. For the coarse grid, the particle-weighted time-averaged vertical particle velocity maps parallel to the $x-z$ plane are given in Figure 5, and for the $x-y$ plane in Figure 6. The slice was $5 \mathrm{~mm}$ thick and located along the center of the bed for the vertical images and at $22 \mathrm{~mm}$ above the distributor for the horizontal images. From visual observation, it was apparent that the velocity maps for each void fraction scheme were in qualitative agreement 
with the experimental data, with the bubbles ascending along the center of the bed, regardless of which void fraction scheme was used (see Figure S3 in the Supporting Information for a time series of images). The experimental measurements show some asymmetry in the location and shape of the bubbling region, where the particles have high upward velocities. The cause of asymmetry of the bubbling region in the experiment was not clear, but may arise from slight imperfections in the symmetry of the experimental apparatus. The simulations predicted that the central bubbling region was wider and more circular than what was recorded by the experiment. The simulations also showed higher particle velocities close to the distributor than was seen experimentally; no downwards particle velocities were observed near the distributor in the coarse grid simulations.

The relative discrepancies between the horizontal particle velocity maps obtained using the void fraction schemes were found using Equation 12 (shown in Table S3 in the Supporting Information). The DPVM, diffusive and satellite point schemes all yield very similar time averaged particle velocity maps with discrepancies typically $<1 \%$. However, the PCM void fraction scheme resulted in larger discrepancies of around 3\% when compared with the other void fraction schemes. The higher discrepancy for the PCM scheme was due to the slight offset in the high-velocity bubbling region of the PCM velocity map compared to the other maps. These results indicate that there is little difference between the void fraction schemes when using a coarse grid, and suggest any of the methods are suitable for simulating the gas-solid flow in this fluidized bed qualitatively.

A quantitative comparison of the experimental and simulated coarse grid velocity data is given in Figure 7. Velocity profiles obtained from three separate experiments are shown in Figure 7(b) and indicate slight differences in the maximum velocity and width of the high-velocity region, 
but overall confirm the reproducibility of the experimental measurements. The measurement uncertainty is indicated by the dashed lines in Figure 7(a) and is approximately $0.02 \mathrm{~m} / \mathrm{s}$. The simulation results in Figure 7(a) show the same trend as the experiments with high velocity in the center of the bed that increases with height above the distributor. As seen in Figure 7(b), close to the vertical walls, the simulations were in good agreement with the experimental data. However, the central bubbling region in the simulations was wider when compared to the experiment, the simulations did not predict negative velocity near the base of the bed, and the velocity between $z=20 \mathrm{~mm}$ and $z=30 \mathrm{~mm}$ was higher than what was observed experimentally. The discrepancies in the vertical maps (shown in Table S4 in the Supporting Information) were generally between $15 \%$ and $20 \%$, and the discrepancies between the simulated and experimental velocity maps along the horizontal slice were between $30 \%$ and $45 \%$ for all void fraction schemes. It was of interest that the error in the PCM results was around the lowest of all of the schemes; this observation was attributed to the offset in the bubbling region of the PCM simulations coinciding with the offset bubbling region from the experiments. It is unlikely that these results would generalize to all conditions, owing to the error in the local void fraction simulations seen in Figure 3. The large error in the horizontal slices arises from differences in the bed expansion and width of the central upward moving particle region. Other studies have found that the behavior of bubbling fluidized beds was not sensitive to the choice of contact force scheme ${ }^{39}$ or contact parameters ${ }^{28}$. Here, we find that all void fraction schemes give similar results, indicating that this discrepancy is unlikely to be due to errors in the void fraction calculation either. Therefore, the effect of refining the fluid grid was investigated.

The particle-weighted time averaged vertical velocities for the fine grid simulations are given in Figure 8. For both tested void fraction schemes, the fine grid simulations predict less regular 
bubbles (see Figure S4 in Supporting Information) and were able to predict the negative particle velocity at the base of the system and the magnitude of the velocity in the center of the bed. Jets due to the inlet holes were more prominent in the simulation than in the experiment, since a cuboidal cell with a side length of $\sim 1.75 \mathrm{~mm}$ was not a perfect representation of a $1 \mathrm{~mm}$ diameter hole. Thus, it is likely that the discrepancy arises in part from insufficient resolution of the inlet geometry, even with the fine grid simulations. To test this theory further, a very fine grid was used, where the cell size was $1.3 d_{p}$. Figures S4-S6 (shown in the Supporting Information) demonstrated that the behavior of this system was qualitatively similar to the fine grid simulations, however the quantitative agreement with the experiment was inferior. These results are consistent with the findings of Peng et al. ${ }^{5}$ and Boyce et al. ${ }^{6}$, who both identified problems in CFD-DEM simulations when the cell size becomes too small. These errors were attributed to a breakdown of the volume averaging assumptions used to derive the fluid equations.

Figure 9(a) illustrates the effect that the enhanced inlet boundary condition had upon the vertical profile of the particle velocity. For $z<25 \mathrm{~mm}$, the fine grid was in excellent agreement with the experiment. Compared to the equivalent coarse grid simulations, the fine grid simulations reduced the discrepancy with the experiment from $\sim 15 \%$ to $\sim 10 \%$ in the vertical images and from $\sim 30 \%$ to $\sim 10 \%$ in the horizontal images. Figure 9 (b) shows that the fine grid predicted a narrower high velocity region near the center, and the maximum velocity is closer to the experiment compared to the coarse grid. These simulations accurately represent the experimental measurements in the vicinity of the distributor, indicating that it is essential to model the gas inlet with a sufficiently fine resolution to capture gas flow accurately. Fine grid simulations were also performed using a uniform inlet (see Figures S8 and S9 in the Supporting Information). These simulations exhibited similar features to the coarse grid simulations such as 
positive velocity at the base of the bed, while the horizontal profile $22 \mathrm{~mm}$ above the base was similar to the fine grid with the realistic inlet. Hence the large error in the coarse grid horizontal maps was reduced by increasing the grid resolution. At heights greater than $22 \mathrm{~mm}$ above the distributor, the fine grid simulation with the uniform inlet closely matches the fine grid simulation with the realistic inlet, while the coarse grid simulations differ significantly. These results suggest that the bubbles are not adequately represented when using the coarse grid. Therefore we suggest that it is necessary for the fluid grid to be sufficiently refined that it can capture the boundary conditions and flow features such as bubbles accurately, and that this may require cells as small as $1.6 d_{p}$. The results also highlight the need for accurate void fraction schemes in these situations, where the PCM method was ineffective.

It is interesting to note that even with the improved representation of the inlet, the predicted velocity above $z=25 \mathrm{~mm}$ was up to $0.1 \mathrm{~m} / \mathrm{s}$ below that of the experiment. As already noted, further refinement of the grid did not improve the agreement with the experiments. Therefore, we also considered the effect of non-sphericity of the particles. The effect of non-sphericity was tested using the effective particle diameter approach ${ }^{40}$. In these simulations, the particle diameter used to calculate the voidage was set to $1.00 \mathrm{~mm}$, such that the voidage at minimum fluidization matched the experiment. The diameter used to calculate the drag then was set to $0.7 \mathrm{~mm}$ such that the simulated minimum fluidization velocity matched the experimental value. Using this effective diameter approach, there was improved agreement with the experimental velocity profile for the coarse grid above the inlet (shown in Figure S7 in the Supporting Information). For the fine grid, the flow near the inlet was represented accurately, as in the conventional simulations, however the velocity was over-estimated elsewhere. These results support previous work suggesting that the gas-solid interaction is not correctly estimated using standard drag 
models $^{41-43}$. For example, the Beetstra drag model does not include the increased drag force experienced by particles due to relative motion of their neighbors ${ }^{41,42}$.

\section{CONCLUSIONS}

A square bubbling fluidized bed was modeled using the CFD-DEM multiphase flow simulation method with several different void fraction schemes at three levels of grid refinement. For the coarse grid, all void fraction schemes produced void fraction maps that were in good agreement with the exact void fraction. Under these conditions, the choice of void fraction scheme did not have an appreciable impact upon the results of the simulation, however there was an error of approximately $30 \%$ in the quantitative predictions of the particle velocity in these cases. The inlet geometry was able to be modeled more accurately by using a fine grid with a cell size of $1.6 d_{p}$. The particle velocity maps obtained from the fine grid simulations were in good qualitative and quantitative agreement with the experiment, providing velocity maps with a relative error of only $10 \%$ for both void fraction schemes tested with the fine grid. It was also found that the PCM was unsuitable for calculating the void fraction with such a refined fluid grid, in agreement with previous research. ${ }^{5,6}$ Furthermore, if the cell size was reduced below $1.6 d_{p}$, the quantitative agreement of the simulations and the experiments worsened.

In conclusion, we find that the PCM approach is only valid for large cell sizes, consistent with the recommendation of Peng et al. ${ }^{5}$. All other approximate void fraction schemes were able to produce similar void fraction fields and velocity fields in similar computational time, provided that the length scale over which the void fraction is distributed is approximately equal to the diameter of the particles. We suggest that if run time is critical, the diffusive scheme is attractive, but in most cases the corrected cube method proposed by Khawaja et al. is likely 
preferable. Finally, it is important to consider the relevant dimensions of the fluid phase cells, if CFD-DEM is to become established as a design tool in engineering.

\section{ACKNOWLEDGEMENTS}

The authors would like to acknowledge the National Energy Technology Laboratory (NETL) of the US Department of Energy for supplying the MFIX CFD-DEM software used in this work. The authors would like to acknowledge S. Strobl, A. Formella, and T. Pöschel for the C++ code used in the exact voidage calculation. DAC would like to acknowledge the financial support of the UC Doctoral Scholarship.

\section{SUPPORTING INFORMATION}

Details of the model equations

Table data and analysis of computation time

Figures of pressure fluctuation profiles, particle velocity frequency distributions, and local mean velocity profiles for simulations using effective diameters to approximate non-sphericity

Time averaged velocity maps for fine grid uniform inlet simulations, and instantaneous void fraction and velocity maps of bubble growth at grid resolution levels of $3.5 d_{p}, 1.6 d_{p}$ and $1.3 d_{p}$

Table data for computation time, simulation parameters, deviations form analytical voidage maps, deviations between simulated horizontal velocity maps, and deviations from the average velocity map of the experiment 


\section{REFERENCES}

(1) Rüdisüli, M.; Schildhauer, T. J.; Biollaz, S. M. A.; Van Ommen, J. R. Scale-up of bubbling fluidized bed reactors - A review. Powder Technol. 2012, 217, 21-38.

(2) van der Hoef, M. A.; van Sint Annaland, M.; Kuipers, J. A. M. Computational fluid dynamics for dense gas-solid fluidized beds: A multi-scale modeling strategy. Chem. Eng. Sci. 2004, 59, 5157-5165.

(3) Freireich, B.; Kodam, M.; Wassgren, C. An exact method for determining local solid fractions in discrete element method simulations. AlChE J. 2010, 56, 3036-3048.

(4) Anderson, T. B.; Jackson, R. A Fluid Mechanical Description of Fluidized Beds. Ind. Eng. Chem. Fundam. 1967, 6, 527-539.

(5) Peng, Z.; Doroodchi, E.; Luo, C.; Moghtaderi, B. Influence of void fraction calculation on fidelity of CFD-DEM simulation of gas-solid bubbling fluidized beds. AlChE J. 2014, 60, 20002018.

(6) Boyce, C. M.; Holland, D. J.; Scott, S. A.; Dennis, J. S. Limitations on Fluid Grid Sizing for Using Volume-Averaged Fluid Equations in Discrete Element Models of Fluidized Beds. Ind. Eng. Chem. Res. 2015, 54, 10684-10697.

(7) Sun, R.; Xiao, H. Diffusion-based coarse graining in hybrid continuum-discrete solvers: Theoretical formulation and a priori tests. Int. J. Multiphase Flow 2015, 77, 142-157.

(8) Tsuji, Y.; Kawaguchi, T.; Tanaka, T. Discrete particle simulation of two-dimensional fluidized bed. Powder Technol. 1993, 77, 79-87.

(9) Sun, R.; Xiao, H. Diffusion-based coarse graining in hybrid continuum-discrete solvers: Applications in CFD-DEM. Int. J. Multiphase Flow 2015, 72, 233-247. 
(10) Strobl, S.; Formella, A.; Pöschel, T. Exact calculation of the overlap volume of spheres and mesh elements. J. Comput. Phys. 2016, 311, 158-172.

(11) NETL, MFIX 2015-1 User Guide. 2015.

(12) Link, J. M.; Cuypers, L. A.; Deen, N. G.; Kuipers, J. A. M. Flow regimes in a spout-fluid bed: A combined experimental and simulation study. Chem. Eng. Sci. 2005, 60, 3425-3442.

(13) Khawaja, H.; Scott, S.; Virk, M.; Moatamedi, M. Quantitative analysis of accuracy of voidage computations in CFD-DEM simulations. J. Comput. Multiphase Flows 2012, 4, 183192.

(14) Zhu, H. P.; Yu, A. B. Averaging method of granular materials. Phys. Rev. E: Stat., Nonlinear, Soft Matter Phys. 2002, 66, 021302/1-021302/10.

(15) Fries, L.; Antonyuk, S.; Heinrich, S.; Palzer, S. DEM-CFD modeling of a fluidized bed spray granulator. Chem. Eng. Sci. 2011, 66, 2340-2355.

(16) Peng, Z.; Moghtaderi, B.; Doroodchi, E. A modified direct method for void fraction calculation in CFD-DEM simulations. Adv. Powder Technol. 2016, 27, 19-32.

(17) Gui, N.; Fan, J. R.; Luo, K. DEM-LES study of 3-D bubbling fluidized bed with immersed tubes. Chem. Eng. Sci. 2008, 63, 3654-3663.

(18) Radl, S.; Gonzales, B.; Goniva, C.; Pirker, S., State of the Art in Mapping Schemes for Dilute and Dense Euler-Lagrange Simulations, in 10th International Conference on CFD in Oil \& Gas, Metallurgical and Process Industries. 2014: Trondheim, Norway. p. 1 - 9.

(19) Hobbs, A. Simulation of an aggregate dryer using coupled CFD and DEM methods. Int. J. Comput. Fluid Dyn. 2009, 23, 199-207. 
(20) Boyce, C. M.; Holland, D. J.; Scott, S. A.; Dennis, J. S. Novel fluid grid and voidage calculation techniques for a discrete element model of a 3D cylindrical fluidized bed. Comput. Chem. Eng. 2014, 65, 18-27.

(21) Holland, D. J.; Müller, C. R.; Dennis, J. S.; Gladden, L. F.; Sederman, A. J. Spatially resolved measurement of anisotropic granular temperature in gas-fluidized beds. Powder Technol. 2008, 182, 171-181.

(22) Syamlal, M.; Rogers, W.; O'Brien, T. J., MFIX documentation theory guide. 1993.

(23) Garg, R.; Galvin, J.; Li, T.; Pannala, S. Open-source MFIX-DEM software for gas-solids flows: Part I-Verification studies. Powder Technol. 2012, 220, 122-137.

(24) Musser, J.; Choudhary, A., MFIX Documentation Volume 3: Verification and Validation

Manual. 2015.

(25) Li, T.; Rogers, W. A.; Syamlal, M.; Dietiker, J.-F.; Musser, J.; Shahnam, M.; Rabha, S. The NETL MFiX Suite of multiphase flow models: A brief review and recent applications of MFiX-TFM to fossil energy Technologies. Chem. Eng. Sci. 2017, 169, 259-272.

(26) Li, T.; Garg, R.; Galvin, J.; Pannala, S. Open-source MFIX-DEM software for gas-solids flows: Part II — Validation studies. Powder Technol. 2012, 220, 138-150.

(27) Gopalakrishnan, P.; Tafti, D. Development of parallel DEM for the open source code MFIX. Powder Technol. 2013, 235, 33-41.

(28) Müller, C. R.; Scott, S. A.; Holland, D. J.; Clarke, B. C.; Sederman, A. J.; Dennis, J. S.; Gladden, L. F. Validation of a discrete element model using magnetic resonance measurements. Particuology 2009, 7, 297-306.

(29) Ye, M.; van der Hoef, M. A.; Kuipers, J. A. M. From discrete particle model to a continuous model of Geldart a particles. Chem. Eng. Res. Des. 2005, 83, 833-843. 
(30) Beetstra, R.; Van Der Hoef, M. A.; Kuipers, J. A. M. Drag force of intermediate reynolds number flow past mono- And bidisperse arrays of spheres. AlChE J. 2007, 53, 489-501.

(31) Shäfer, J.; Dippel, S.; Wolf, D. Force schemes in simulations of granular materials. J. Phys. I 1996, 6, 5-20.

(32) Silbert, L. E.; Ertaş, D.; Grest, G. S.; Halsey, T. C.; Levine, D.; Plimpton, S. J. Granular flow down an inclined plane: Bagnold scaling and rheology. Phys. Rev. E 2001, 64, 051302.

(33) Garg, R.; Galvin, J.; Li, T.; Pannala, S., Documentation of open-source MFIX-DEM software for gas-solids flows. 2012.

(34) Deen, N. G.; Van Sint Annaland, M.; Van der Hoef, M. A.; Kuipers, J. A. M. Review of discrete particle modeling of fluidized beds. Chem. Eng. Sci. 2007, 62, 28-44.

(35) Boyce, C. M.; Holland, D. J.; Scott, S. A.; Dennis, J. S. Adapting data processing to compare model and experiment accurately: A discrete element model and magnetic resonance measurements of a 3d cylindrical fluidized bed. Ind. Eng. Chem. Res. 2013, 52, 18085-18094.

(36) Johnson, N. L. Systems of frequency curves generated by methods of translation. Biometrika 1949, 36, 149-176.

(37) Kloss, C.; Goniva, C.; Hager, A.; Amberger, S.; Pirker, S. Models, algorithms and validation for opensource DEM and CFD-DEM. Prog. Comput. Fluid Dyn. 2012, 12, 140-152.

(38) Hilton, J. E.; Mason, L. R.; Cleary, P. W. Dynamics of gas-solid fluidised beds with nonspherical particle geometry. Chem. Eng. Sci. 2010, 65, 1584-1596.

(39) Di Maio, F. P.; Di Renzo, A. Modelling Particle Contacts in Distinct Element Simulations. Chem. Eng. Res. Des. 2005, 83, 1287-1297. 
(40) Boyce, C. M.; Ozel, A.; Rice, N. P.; Rubinstein, G. J.; Holland, D. J.; Sundaresan, S. Effective particle diameters for simulating fluidization of non-spherical particles: CFD-DEM models vs. MRI measurements. AlChE J. 2017, 63, 2555-2568.

(41) Kriebitzsch, S. H. L.; Van Der Hoef, M. A.; Kuipers, J. A. M. Fully resolved simulation of a gas-fluidized bed: A critical test of DEM models. Chem. Eng. Sci. 2013, 91, 1-4.

(42) Tang, Y.; Peters, E. A. J. F.; Kuipers, J. A. M. Direct numerical simulations of dynamic gas-solid suspensions. AlChE J. 2016.

(43) Radl, S.; Sundaresan, S. A drag model for filtered Euler-Lagrange simulations of clustered gas-particle suspensions. Chem. Eng. Sci. 2014, 117, 416-425. 


\section{Figures}

(a)

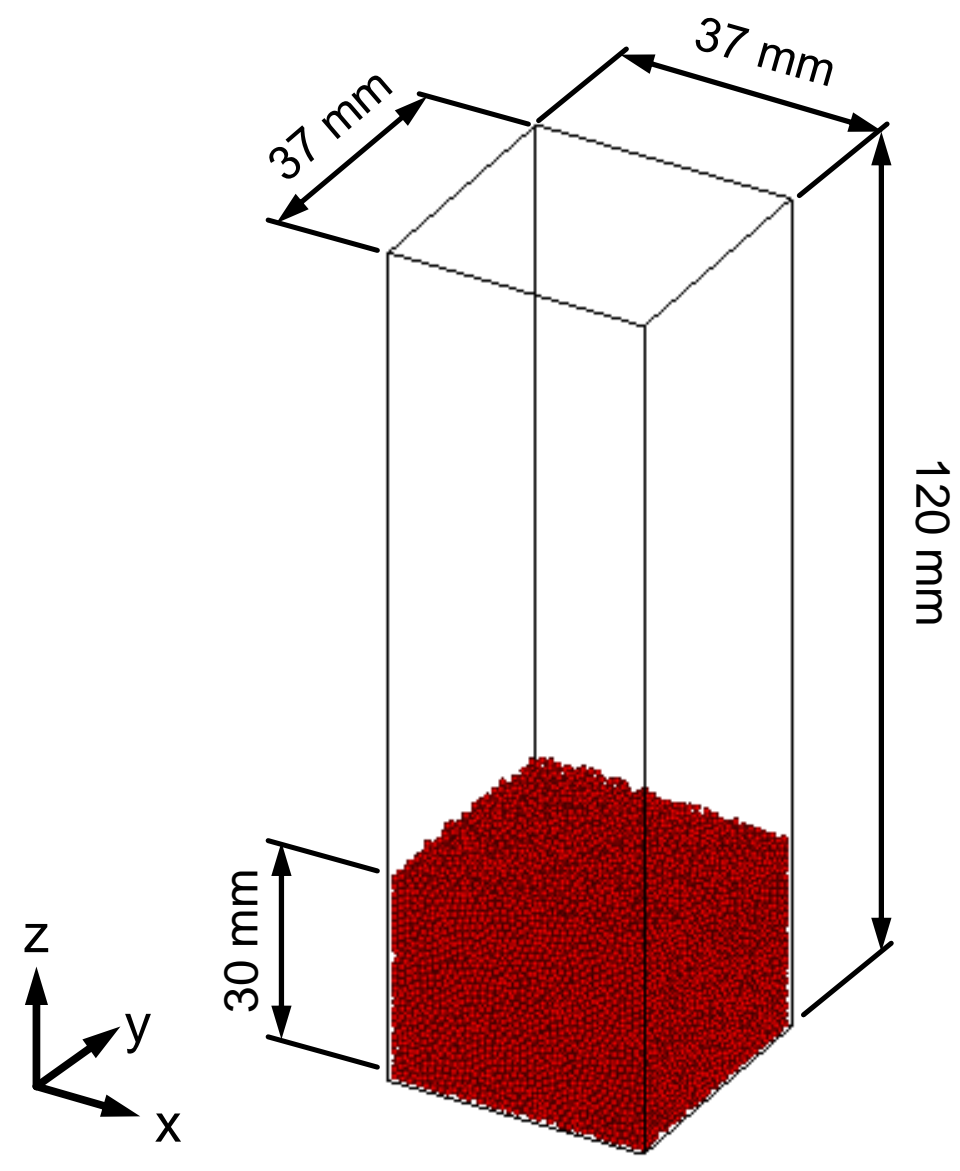

(b)

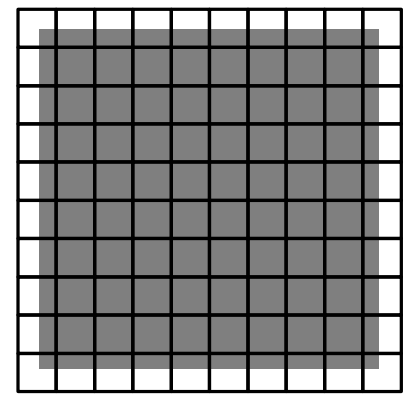

(c)

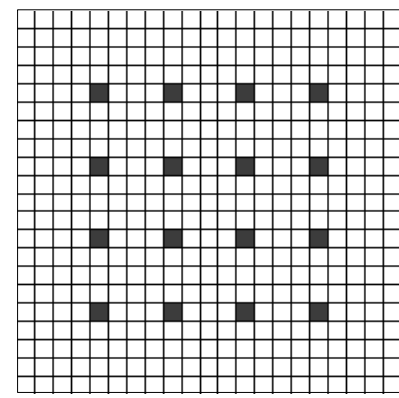

(d)


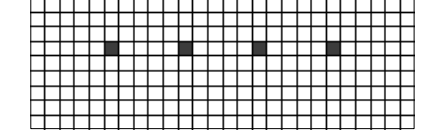

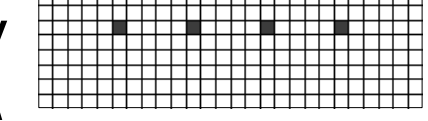

Figure 1. (a) Schematic diagram of the square fluidized bed used in the simulations. The domain boundaries are denoted by the black outlines. (b) Uniform inlet boundary condition and fluid grid used for the coarse grid simulation. (c) Drilled plate inlet boundary condition and fluid grid used for fine grid simulation. (d) Drilled plate inlet boundary condition and fluid grid used for very fine grid simulation. The gas flow regions are denoted by dark grey. 

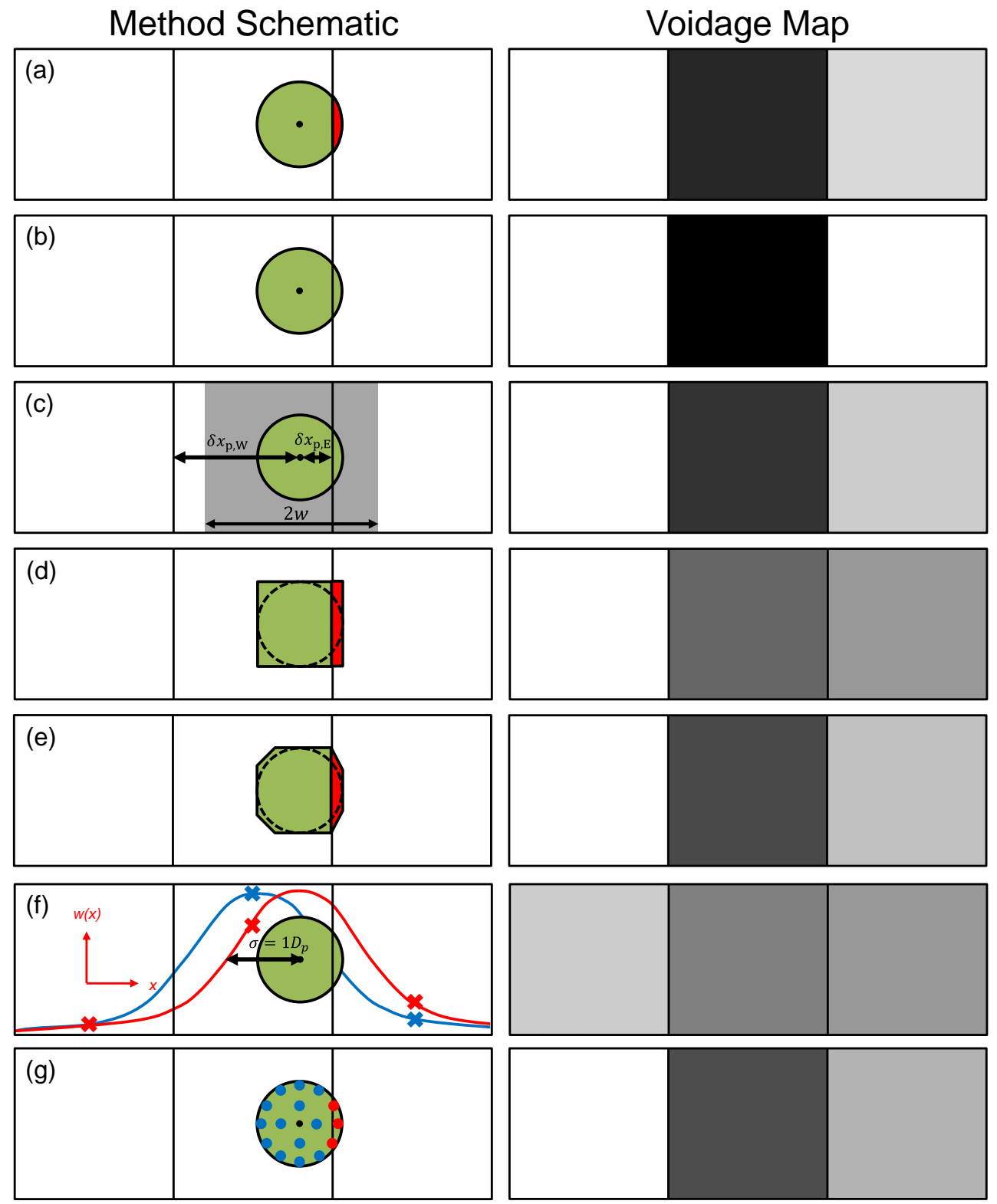

Figure 2. (Left) diagram illustrating how each void fraction scheme determines particle volume fractions and (right) representations of the particle volume fraction map for: (a) analytical method, (b) PCM, (c) MFIX DPVM, (d) cube DPVM, (e) corrected cube DPVM. (f) statistical kernel method using a Gaussian distribution with a standard deviation of $1 d_{p}$ (red line). The equivalent PCM initialized diffusion method is shown by the blue line. (g) Satellite point method. 

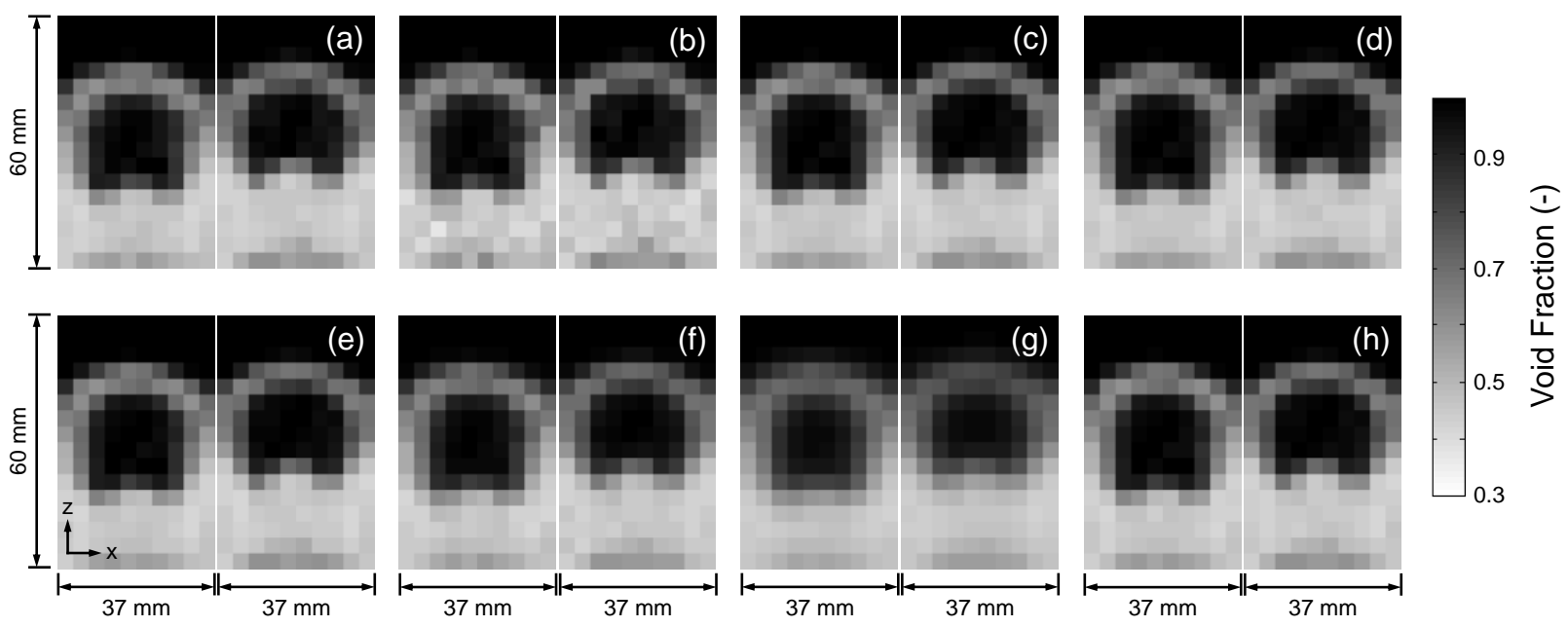

Figure 3. Instantaneous void fraction maps for two time steps $0.01 \mathrm{~s}$ apart obtained using the coarse fluid grid. The map was located in the $x-z$ plane along a central slice in the $y$-direction for a snapshot of particles using: (a) exact, (b) PCM, (c) MFIX DPVM, (d) Cube DPVM, (e) Corrected cube DPVM, (f) diffusive scheme with $\sigma=1 d_{p}$, (g) diffusive scheme with $\sigma=2 d_{p}$, (h) satellite point method. Particle positions were the same for all void fraction images, only the method used to calculate the void fraction was changed. The grid dimensions were 10 cells $\times 10$ cells $\times 32$ cells. 

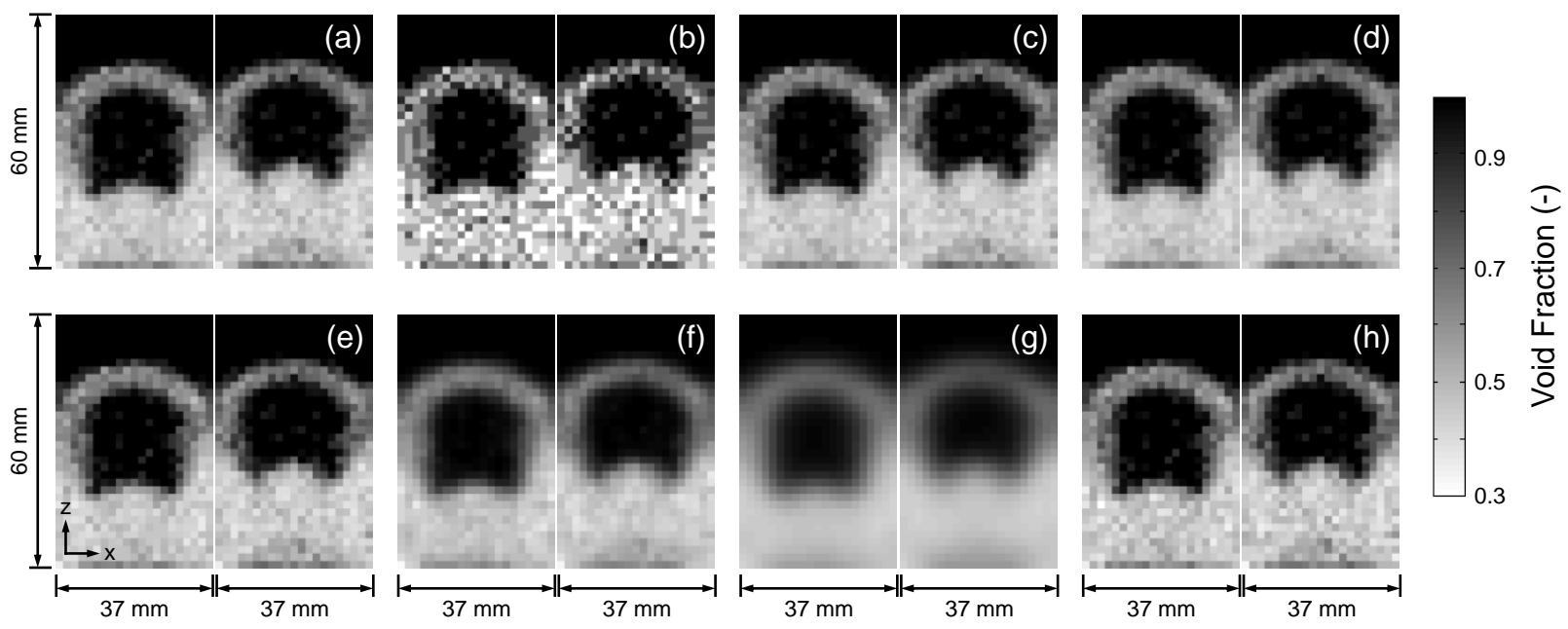

Figure 4. Instantaneous void fraction maps for two time steps $0.01 \mathrm{~s}$ apart obtained using the refined fluid grid. The map was located in the $x-z$ plane along a central slice in the $y$-direction for a snapshot of particles using: (a) exact, (b) PCM, (c) MFIX DPVM, (d) Cube DPVM, (e) Corrected cube DPVM, (f) diffusive scheme with $\sigma=1 d_{p}$, (g) diffusive scheme with $\sigma=2 d_{p}$, (h) satellite point method. Particle positions were the same for all void fraction images, only the method used to calculate the void fraction was changed. The grid dimensions were 21 cells $\times 21$ cells $\times 68$ cells. 


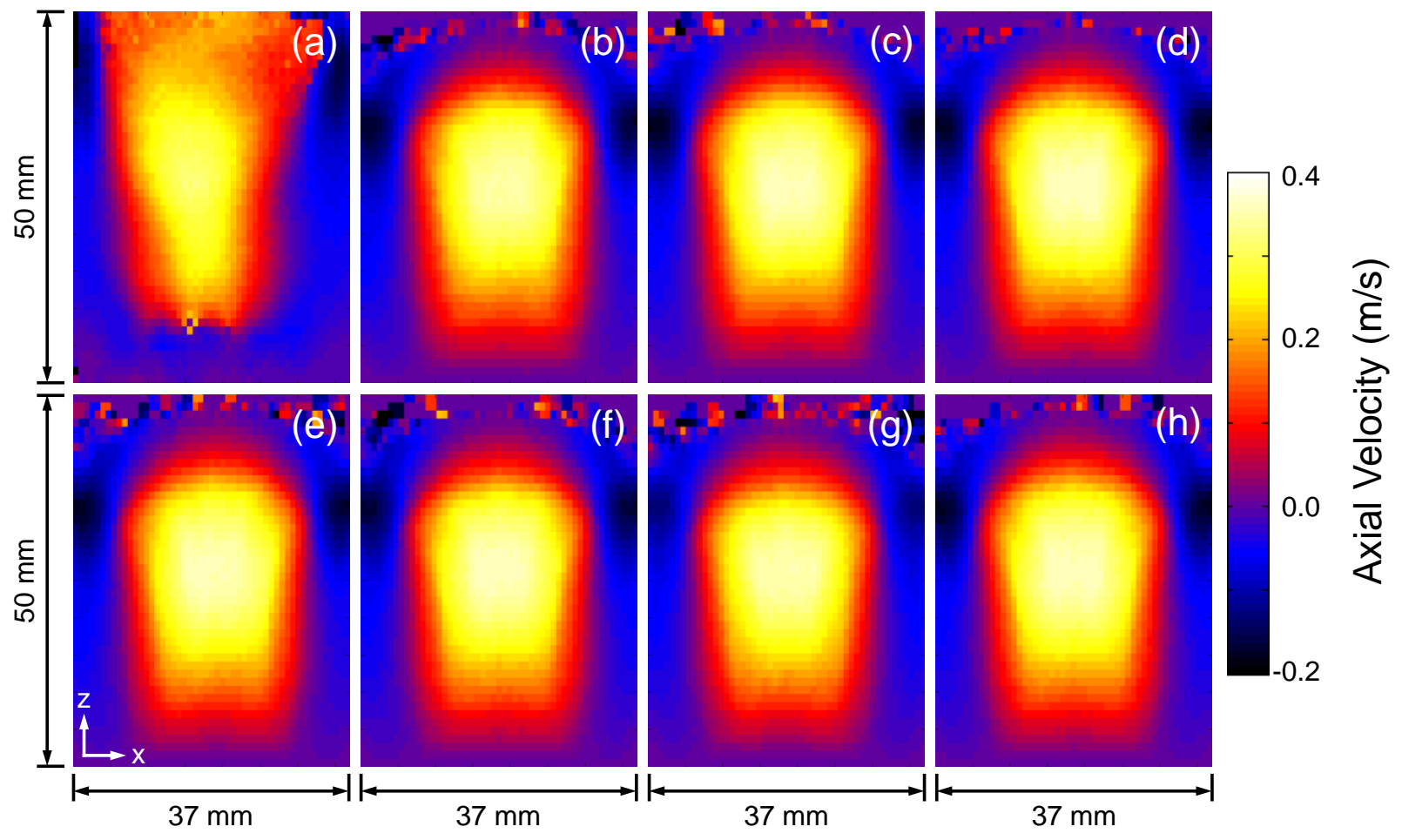

Figure 5. Particle-weighted time-averaged maps of the vertical component of the particle velocity along a central slice parallel to the $x$-z plane for: (a) experiment, (b) particle-centered method, (c) MFIX DPVM, (d) cube DPVM, (e) corrected cube DPVM, (f) diffusive scheme with $\sigma=1 d_{p}$, (g) diffusive scheme with $\sigma=2 d_{p}$, (h) satellite point method. Simulations were performed using the coarse fluid grid. The resolution of each image is $0.7 \mathrm{~mm}(x) \times 1.09 \mathrm{~mm}(z)$. 




Figure 6. Particle-weighted time-averaged maps of the vertical component of the particle velocity along a horizontal slice centered at $z=22 \mathrm{~mm}$ for: (a) experiment, (b) particle-centered method, (c) MFIX DPVM, (d) cube DPVM, (e) corrected cube DPVM, (f) diffusive scheme with $\sigma=1 d_{p}$, (g) diffusive scheme with $\sigma=2 d_{p}$, (h) satellite point method. Simulations were performed using the coarse fluid grid. The resolution of each image is $0.7 \mathrm{~mm}(x) \times 0.7 \mathrm{~mm}(y)$. 

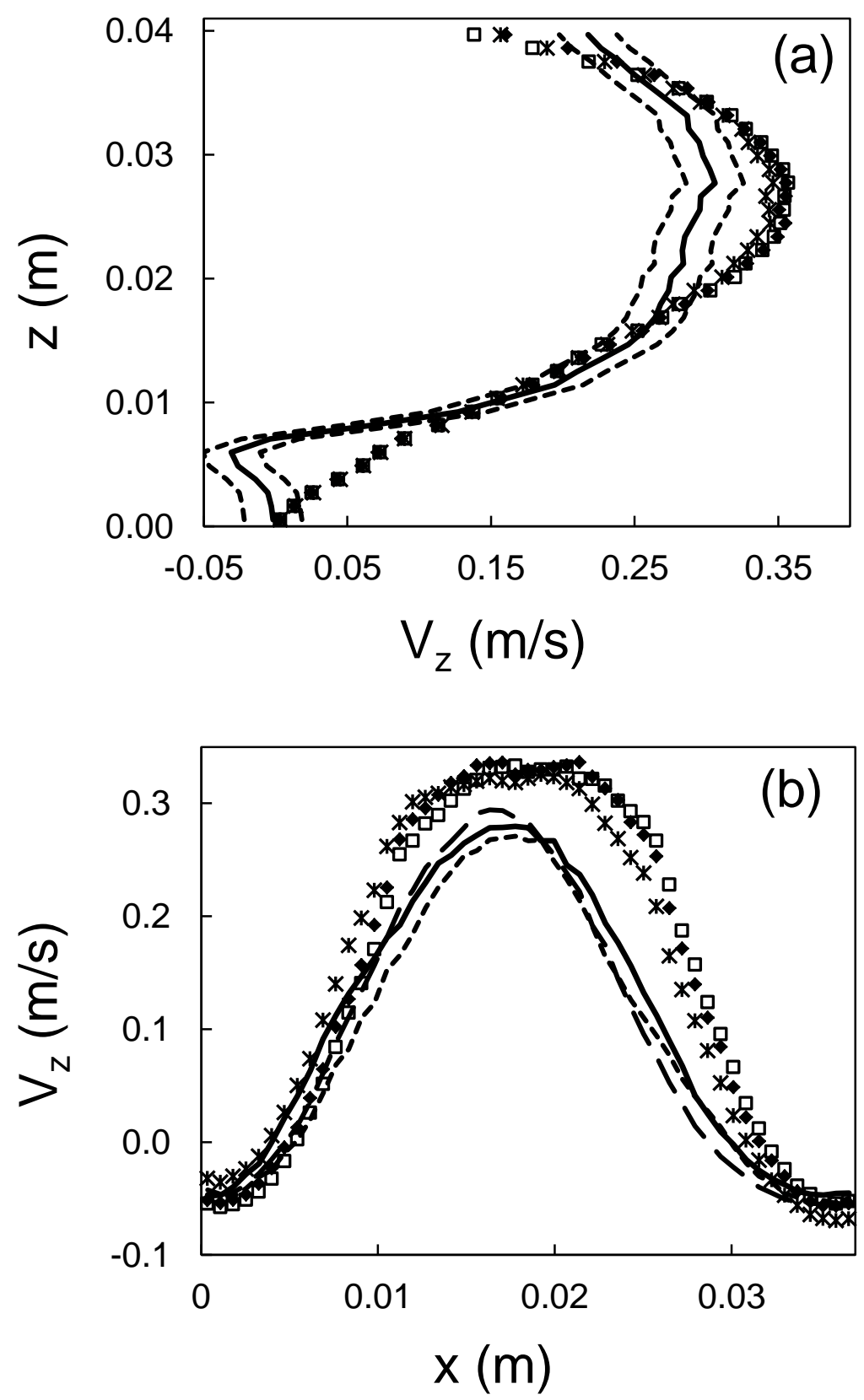

Figure 7. Comparison of the experimental measurement and coarse grid simulations for the particle-weighted time-averaged vertical component of the particle velocity. The profiles are shown along: (a) central axis in the $z$-direction at $x=18.5 \mathrm{~mm}$, and $y=18.5 \mathrm{~mm}$, (b) along the $x$ direction at $y=18.5 \mathrm{~mm}$ and $z=22 \mathrm{~mm}$. (Experiments: lines, PCM: * Corrected cube DPVM:

๑, Diffusive with $\sigma=1 d_{p}$ : $\square$ ) 


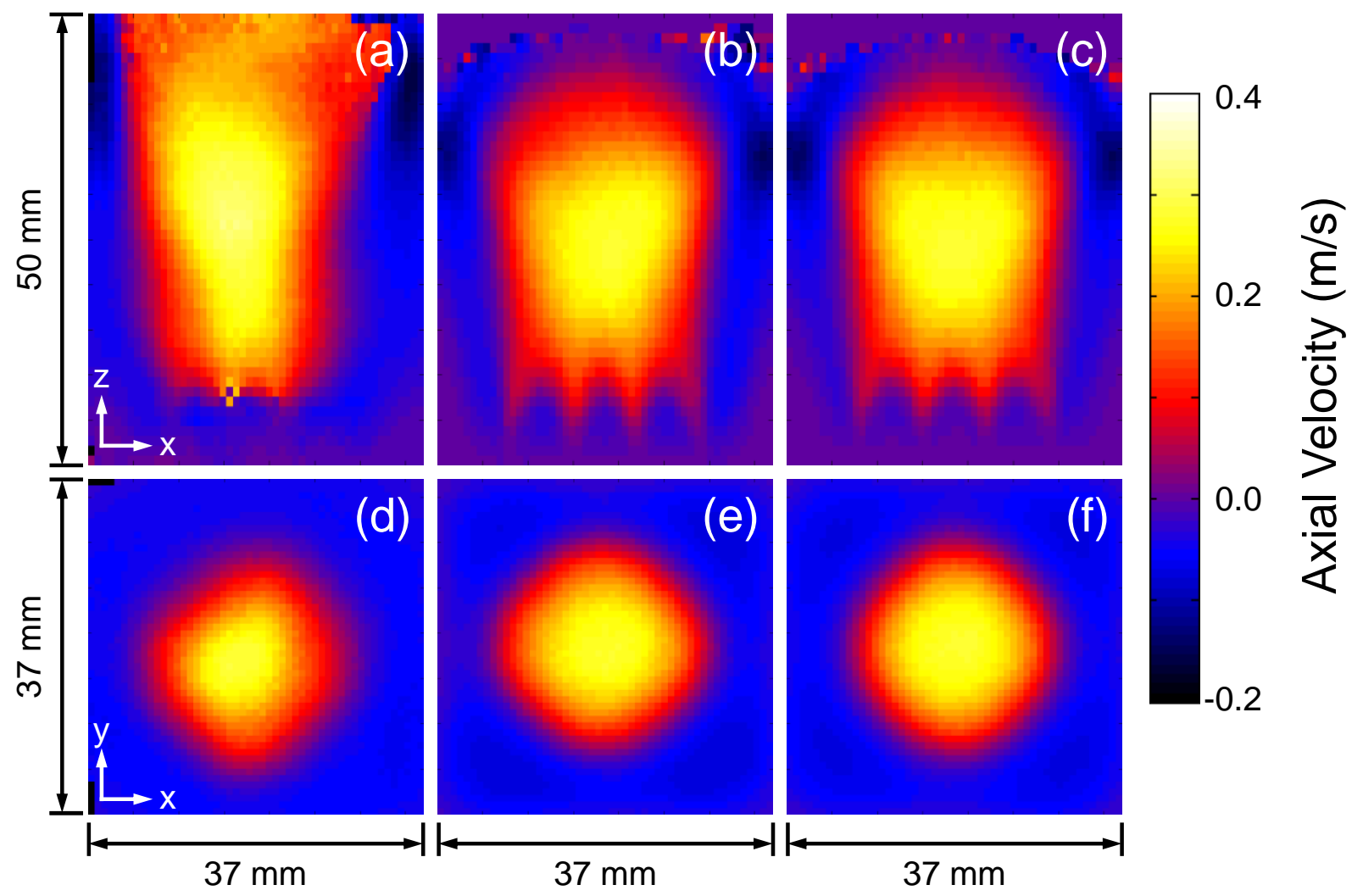

Figure 8. Particle-weighted time-averaged maps of the vertical component of the particle phase velocity along a central slice parallel to the $x$-z plane for: (a) experiment, (b) corrected cube DPVM, (c) diffusive scheme with $\sigma=1 d_{p}$. The resolution of the images along the top row is 0.7 $\mathrm{mm}(x) \times 1.09 \mathrm{~mm}(z)$. Particle-weighted time-averaged vertical particle phase velocity maps along a horizontal slice centered at $z=22 \mathrm{~mm}$ for: (d) experiment, (e) corrected cube DPVM, (f) diffusive scheme with $\sigma=1 d_{p}$. Simulations used the refined fluid grid. The resolution of the images along the lower row is $0.7 \mathrm{~mm}(x) \times 0.7 \mathrm{~mm}(y)$. 

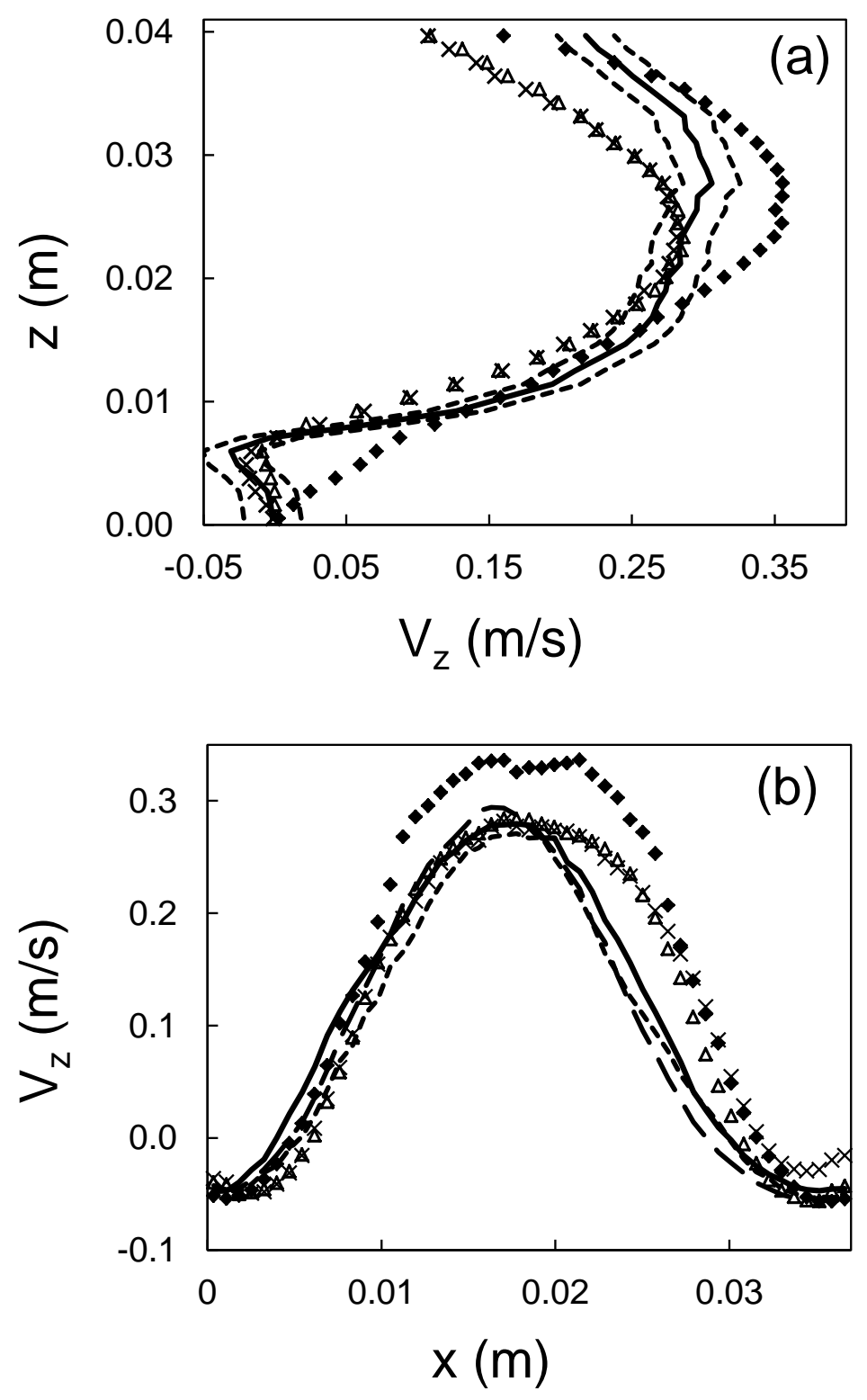

Figure 9. Comparison of the experimental measurement and simulation results for the particleweighted time-averaged vertical component of the particle velocity. The profiles are shown along (a) central axis in the $z$-direction at $x=18.5 \mathrm{~mm}$, and $y=18.5 \mathrm{~mm}$, (b) along the $x$ direction at $y=18.5 \mathrm{~mm}$ and $z=22 \mathrm{~mm}$. (Experiments: lines, Fine grid and corrected cube DPVM: $\times$, Fine grid and diffusive scheme with $\sigma=1 d_{p}: \Delta$, Coarse grid and corrected cube DPVM: 
For Table of Contents Only

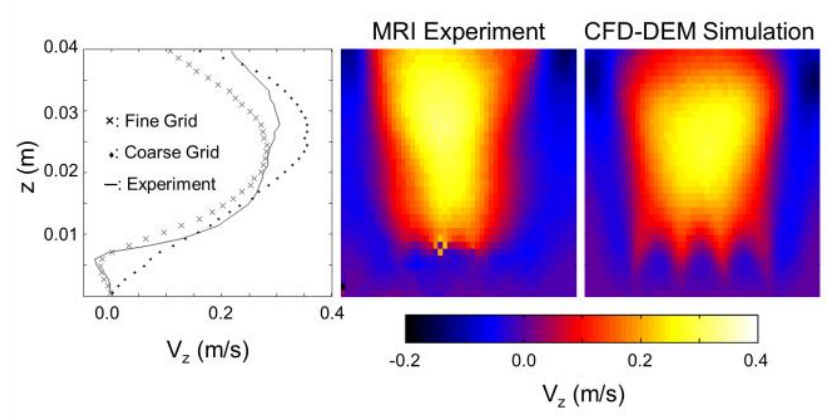

